\title{
The Effect of Sodium Chloride on the Process of Drying of Porous Media
}

\author{
I.M. FiJAe-KirejcZYK*, J.J. MilczAREK, Z. JuRKowski And J. ŻoŁĄDEK \\ National Centre for Nuclear Research (NCBJ), 05-400 Otwock-Świerk, Poland
}

\begin{abstract}
The high temperature drying process of corundum cylinders partially saturated with aqueous solutions of sodium chloride was investigated with neutron imaging technique accompanied with mass and temperature measurements. The statistical analysis of neutron images was applied in order to quantify the time evolution of the process. The substantial slowing down of drying with increasing salt concentration was found. This effect was attributed to increase of viscosity of the solution with increasing salt content as well as to the formation of an outer layer of $\mathrm{NaCl}$ on the sample surface.
\end{abstract}

DOI: $10.12693 /$ APhysPolA.124.1025

PACS: 61.05.Tv, 07.05.Pj, 81.05.Rm

\section{Introduction}

Drying the porous material containing solutions of salts leads to crystallization of salts on the outer surfaces (efflorescence) and within the pores (subflorescence) of the medium [1-4]. These processes have been intensively studied recently because they can produce irretrievable damage of valuable objects (sculptures, buildings) as well as current structures of civil engineering [5]. From a practical point of view the dependence of drying rate on the salt concentration is of importance. In particular an investigation of drying process carried out at elevated temperatures exceeding that of the usual ambient conditions may provide new information interesting both for basic and practical purposes.

In this work the digital thermal neutron radiography was used as a main research tool augmented with traditional gravimetric and temperature measurements. The neutron radiography has been used for at least a decade to study the migration of hydrogen containing liquids in a porous medium. The main advantage of the thermal neutron imaging is provided by very strong incoherent scattering of thermal neutrons by hydrogen nuclei [6-11] and relatively weak thermal neutron interaction with other elements.

Since drying of the capillary-porous objects is a highly non-equilibrium multistage process it is interesting to find effects of salts on the subsequent periods of the process. The different stages of the drying process are conventionally distinguished by different rate of mass loss of the sample $[12,13]$. The first period of very high rate of mass decrease has been named the constant rate period (CRP) due to almost linear decrease of the mass on time. The CRP is followed by two falling rate periods (FRP) which differ in mechanism of water transport from the sample body to the sample surface. For the

\footnotetext{
* corresponding author; e-mail: Izabela.Fijal-Kirejczyk@ncbj.gov.pl
}

second FRP the concept of a drying front separating the dry outer region from the wet central core has been introduced [14, 15]. This phase of drying is also known as the receding front period (RFP) and has been visualized unequivocally with the neutron imaging [8-11].

In this work the experimental results for the process of drying of cylinders made of particulate corundum of two different grain sizes, wetted with aqueous salt solutions of different $\mathrm{NaCl}$ concentrations are presented. The hot air was used as a drying agent, and the lateral side of cylinders was exposed to the air flow. Drying process was observed and recorded via neutron radiography accompanied by on-line measurements of temperature inside the samples and their weight change. For process quantification the global statistical parameters (average brightness and its standard deviation) of the sample neutron images were analysed according to the procedure proven to be useful for description of drying processes of wet cylinders $[8,10,11]$.

\section{Experiments}

The experiments were carried out at the neutron radiography station (NGRS) at the M RI nuclear research reactor of NCBJ [7-11].

The experimental setup was the same for all drying samples. The cylindrical sample was placed inside a vertical tunnel in which $\approx 90^{\circ} \mathrm{C}$ hot air stream was flowing vertically from above with velocity $\approx 2 \mathrm{~m} / \mathrm{s}$. The sample axis was at the distance of $\approx 95 \mathrm{~mm}$ from the flat neutron detector screen. The investigated sample was placed on an aluminum support with its lower end resting at an electronic balance. The sample support incorporated the K-type steel sheathed thermocouple of $0.5 \mathrm{~mm}$ diameter. The thermocouple penetrated the sample vertically from its lower end to the middle. The drying process was observed and recorded with neutron radiography accompanied by on-line measurements of temperature inside the sample and its mass change (Fig. 1). The sample's temperature and mass were acquired every $1 \mathrm{~s}$ and $10 \mathrm{~s}$, respectively, and the neutron radiographs were acquired every $5 \mathrm{~s}$. 


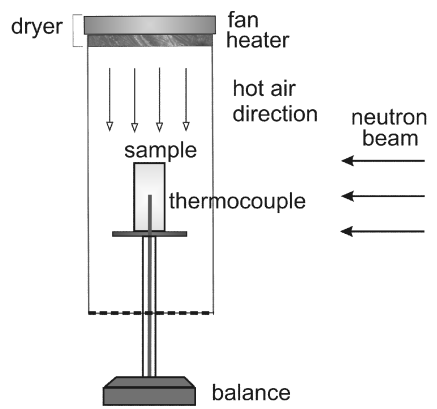

Fig. 1. Diagram of the experimental arrangement.

The experiments were performed on samples prepared from chemically inert particulate electrocorundum (containing more than $99 \%$ of $\mathrm{l}_{2} \mathrm{O}_{3}$ ) saturated with aqueous solutions of sodium chloride. The materials of two different grain size ranges of: $250-300 \mu \mathrm{m}(\mathrm{F} 60)$ and $53-75 \mu \mathrm{m}$ (F220) were studied. Each of them was measured for many different aqueous $\mathrm{NaCl}$ solutions of concentrations ranging from 1 to $26.3 \%$ mass. The cylindrical samples were obtained by extrusion with a diameter of $27 \mathrm{~mm}$ and a height of $\approx 30 \mathrm{~mm}$. Initial moisture content in all samples was $\approx 10 \%$ of mass and wet materials were prepared $\approx 48 \mathrm{~h}$ before experiment.

The neutron flux density at the sample was $\approx 10^{7} \mathrm{n} / \mathrm{cm}^{2} / \mathrm{s}$, and the single frame exposure time was $1.56 \mathrm{~s}$. The projection ratio provided by the optical system was $154 \mu \mathrm{m} /$ pixel, and was determined with the application of a $80 \mathrm{~mm}$ long standard cadmium plate. The image analysis was performed with the software designed for image acquisition: HiPic ver. 9.1 Hamamatsu and Fiji/ImageJ 1.46r (NIH) analytical software packages.

Before image analysis a preprocessing calibration procedure including the correction of pixel brightness for the electronic dark current of the CCD camera, normalization for neutron beam flux fluctuations via an averaged open field neutron radiograph as well as the median filtering were applied. The values of brightness discussed further on in this work are corrected values.

\section{Results}

The determined dependence of the sample mass on time reveals the initial period of fast decrease in water content. This period starts immediately after the initial phase of heating the sample to the drying air temperature and exist independently of the salt concentration (Figs. 2 and 3) and the size of the medium grains. The drying rate at this period decreases with $\mathrm{NaCl}$ concentration.

The plots of the mass on time dependence consist of two distinctly different periods characterized with different decrease rates only for small $\mathrm{NaCl}$ concentration. The presence of different periods of the process was found even less discernible in plots of the time dependence for the temperature inside the sample (Figs. 4 and 5). Only for small salt concentration the CRP and FRP periods can be easily distinguished by the steady temperature

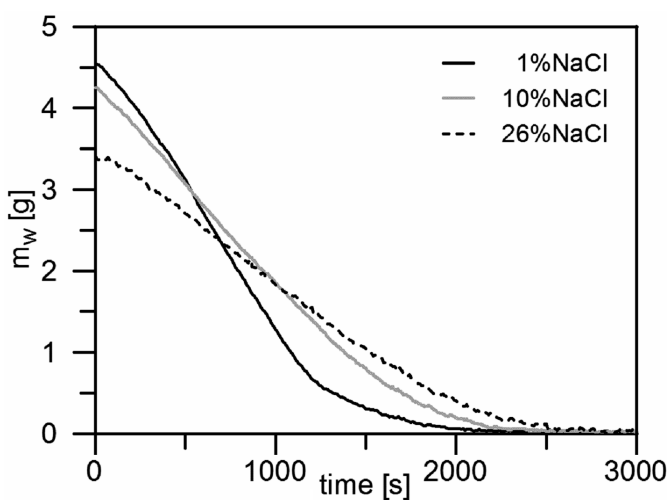

Fig. 2. Examples of mass variation with time for the drying of corundum F60 samples.

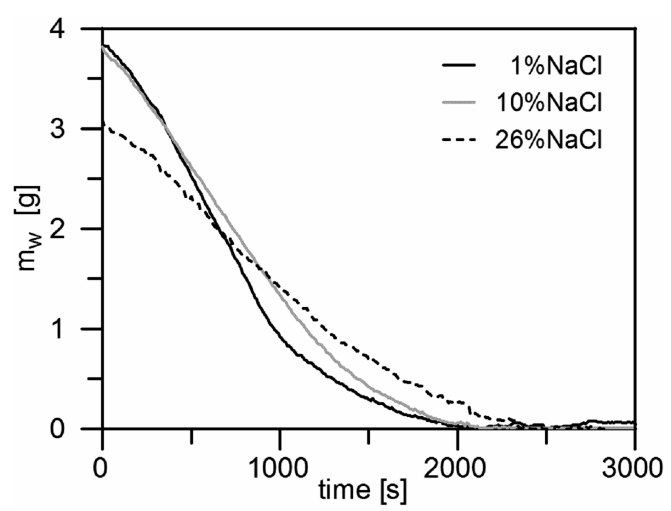

Fig. 3. Examples of mass variation with time for the drying of corundum F220 samples.

intervals separated with discernible kink in temperature vs. time dependence. The marked increase in the slope of temperature vs. time dependence at the transition between CRP and FRP exists only for solutions of small $\mathrm{NaCl}$ content.

The evolution of main qualitative features of neutron radiograms during drying was similar for all samples irre-



Fig. 4. Examples of the temperature dependence on time for drying of corundum F60 samples. 


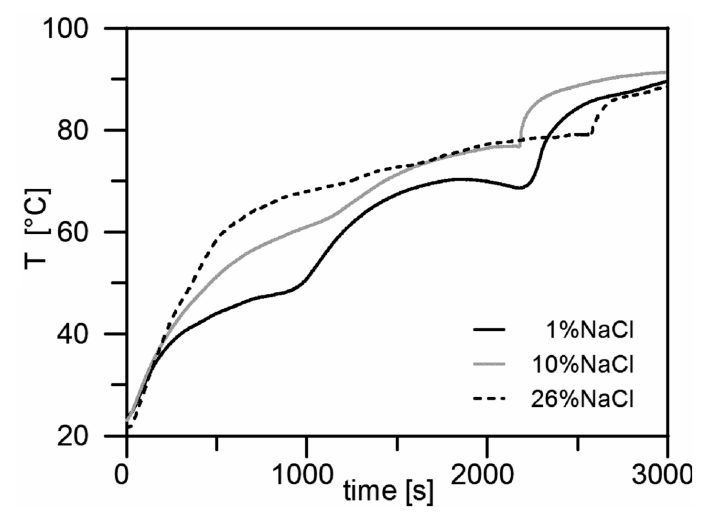

Fig. 5. Examples of the temperature dependence on time for drying of corundum F220 samples.

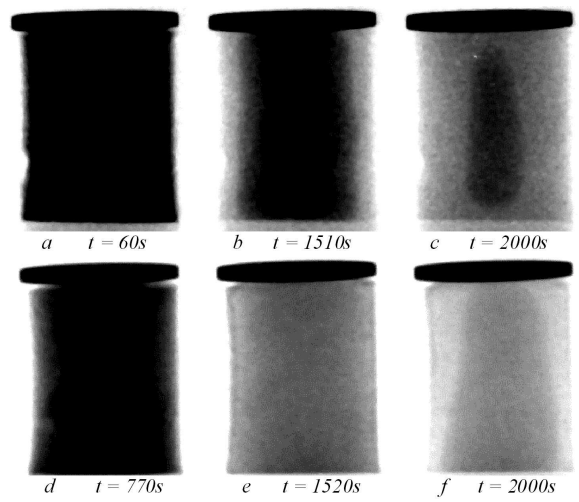

Fig. 6. The time evolution of the corundum F60 sample image during drying for two different $\mathrm{NaCl}$ concentration: ( $\mathrm{a}, \mathrm{b}, \mathrm{c})$ correspond to $1 \% \mathrm{NaCl}$ and the times $60 \mathrm{~s}, 1510 \mathrm{~s}, 2000 \mathrm{~s}$, respectively, and (e,f,g) correspond to $23 \% \mathrm{NaCl}$ and the times $770 \mathrm{~s}, 1520 \mathrm{~s}$, and $2000 \mathrm{~s}$, respectively.

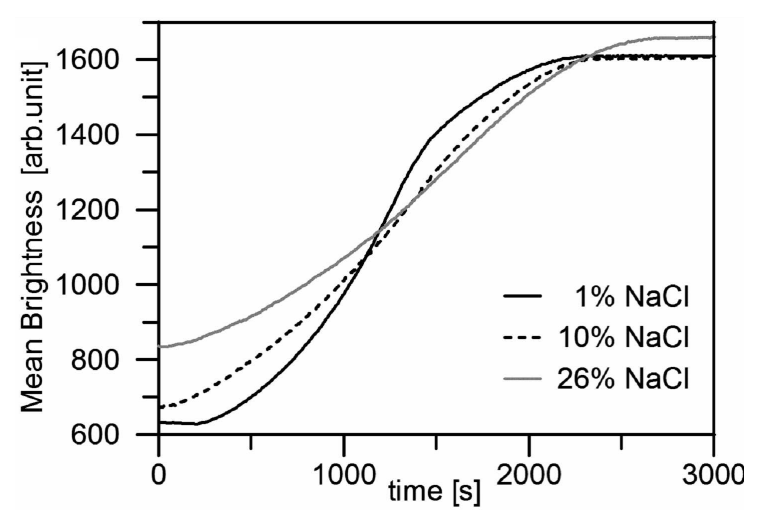

Fig. 7. Examples of average brightness of the neutron images dependence on time for drying of corundum F60 samples. spective of the $\mathrm{NaCl}$ concentration (Fig. 6). The increase of total brightness of the sample image was found for every case investigated (Fig. 7). Moreover, the existence of the inner dark region attributed to the inner wet region limited by a drying front was revealed for the later drying period (Fig. 6b,c and e,f).

\section{Analysis and discussion}

In order to quantify the differences in behavior of samples saturated with solution of different salt concentration the statistical analysis of images was performed. The average brightness and standard deviation of the brightness were calculated with the ImageJ software package and their time evolution was analyzed [8-11]. It was found that the standard deviation of brightness exhibits two maxima when plotted vs. time (Fig. 8). The first of them appeared closer to the beginning of the process when the salt concentration was increasing, whereas the second one shifted towards later times (Fig. 9). This effect was much stronger in the medium made of larger grains (F60) than in the finer grain (F220) material.

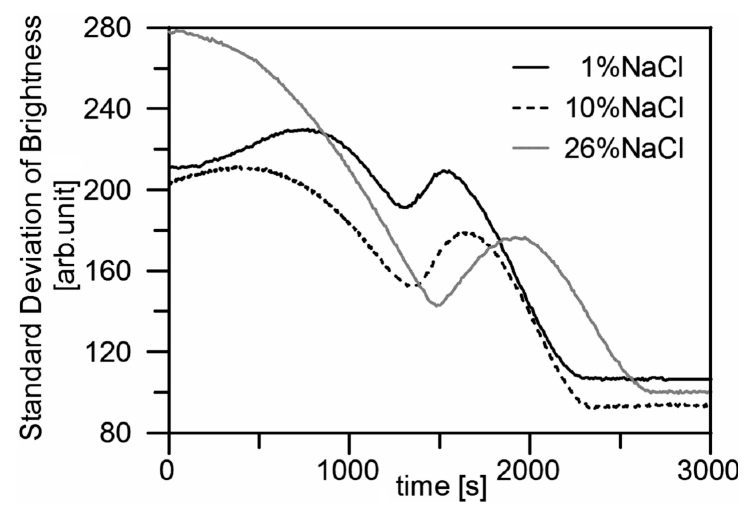

Fig. 8. Examples of the standard deviation of the neutron images dependence on time for drying of corundum F60 samples.

These results show that the drying process was slower for solutions containing larger salt amounts. The physical processes occurring inside drying cylinders and their effects on the neutron radiographs have been described previously within a two-cylinders model of the sample $[9,11]$. Within this model the brightness radial profiles and the standard deviation of brightness have been explained. It has been proven $[9,11]$ that the occurrence of the first maximum has to be attributed to the decreasing contribution of the scattered neutrons component to the image brightness due to decreasing water content. Hence, the observed shift of the first maximum towards the beginning of the drying indicates that the contribution of the scattering decreases with increasing salt concentration. This effect can be explained in terms of the neutron macroscopic cross-section for scattering of aqueous $\mathrm{NaCl}$ solutions. Since the microscopic cross-section for neutron scattering on $\mathrm{Na}$ and $\mathrm{Cl}$ is much less than that of hydrogen, the presence of $\mathrm{NaCl}$ reduces the macroscopic 


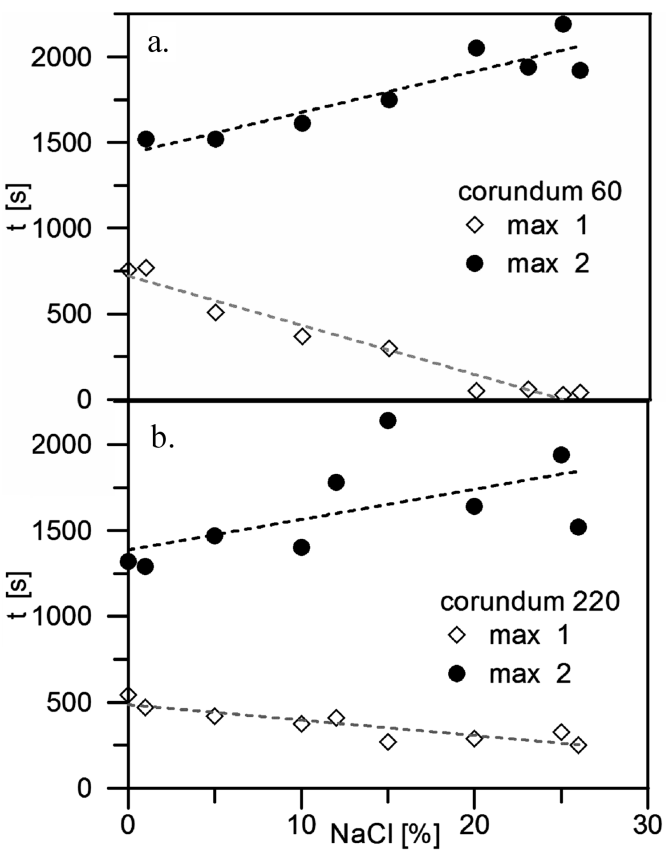

Fig. 9. The dependence of the time positions of the brightness standard deviation maxima on the $\mathrm{NaCl}$ concentrations for (a) F60 and (b) F220 corundum.

neutron cross-section of the solution with respect to that of pure water.

Our previous analysis $[9,11]$ confirmed that the presence of the second maximum in the plot of the standard deviation was due to the development of the limited inner wet region and the motion of the drying front towards centre. The observed shift of the second maximum towards later times with increasing salt concentration was certainly due to the later development and slower motion of the drying front in samples with larger salt content.

The decrease of the drying rate with $\mathrm{NaCl}$ concentration can be explained by two factors. The first one is the increase of the solution viscosity with salt concentration which slows down the capillary flow of the liquid. This effect is important in the CRP period which consists essentially in capillary transport of the liquid. At the later time a partial subflorescence of salt can occur. The subflorescence should decrease the size of pores reducing the flow rate of the liquid. The second contributing factor is the precipitation of salt on the sample surface from which the evaporation proceeds. This efflorescence can block open superficial ends of capillaries reaching the surface reducing in effect the evaporation of water. The outer layer of precipitated salt can also accumulate water for some time yielding lower evaporation rate.

The effect of salt in the wetting solution was not limited to reduction the drying rate. We observed that the salt enhanced the mechanical stability of the samples. One should mention here that the cylinders produced from the corundum saturated with pure water became unstable and fell apart with diminishing water content during drying. Even small content of $\mathrm{NaCl}(1 \%)$ produced samples that not only survived the drying procedure but also stayed intact after post-experimental treatments. Since no change in the samples' sizes was observed it seems that the crystallization of salt in the inter-grain spaces was not significant.

\section{Conclusions}

We found that the increase in salt concentration of the solution slows down the high temperature drying process. Moreover, the differences between the main stages of the drying process seem to disappear with increasing salt concentration mainly due to the reduced rate in the first stage of drying. The effect of salt concentration is much more pronounced for the medium composed of finer grains. There is strong evidence that during drying a layer of salt is formed on the surface of the sample which may be responsible for slowing down of the process.

Moreover, we observed that the presence of salt increases the mechanical strength of the samples. However, the salt crystallization within the sample volume (subflorescence) is not significant since there was no increase in samples size during the drying process.

\section{References}

[1] H. Eloukabi, N. Sghaier, S. Ben Nassrallah, M. Prat, Int. J. Heat Mass Transf. 56, 80 (2013).

[2] H. Eloukabi, N. Sghaier, M. Prat, S. Ben Nassrallah, Chem. Eng. Technol. 34, 1085 (2011).

[3] S. Veran-Tissoires, M. Marcoux, M. Prat, Phys. Rev. Lett. 108, 054502 (2012).

[4] S. Veran-Tissoires, M. Marcoux, M. Prat, Europhys. Lett. 98, 34005 (2012).

[5] M. Schiro, E. Ruiz-Agudo, C. Rodriguez-Navarro, Phys. Rev. Lett. 109, 265503 (2012).

[6] M. Strobl, I. Manke, N. Kardjilov, A. Hilger, M. Dawson, J. Banhart, J. Phys. D: Appl. Phys. 42, 243001 (2009).

[7] I. Fijał-Kirejczyk, J.J. Milczarek, J. Banaszak, A. Trzciński, J. Żołądek, Nukleonika 54, 123 (2009).

[8] I.M. Fijał-Kirejczyk, J.J. Milczarek, J. ŻołądekNowak, Nucl. Instrum. Meth. Phys. Res. A 651, 201 (2011).

[9] I.M. Fijał-Kirejczyk, J.J. Milczarek, J. ŻołądekNowak, F.C. de Beer, M.J. Radebe, G. Nothnagel, Acta Phys. Pol. A 122, 410 (2012).

[10] I.M. Fijał-Kirejczyk, J.J. Milczarek, F.C. de Beer, M.J. Radebe, G. Nothnagel, J. Żołądek-Nowak, Nukleonika 57, 529 (2012).

[11] I.M. Fijał-Kirejczyk, J.J. Milczarek, M.J. Radebe, F.C. de Beer, G. Nothnagel, J. Żołądek-Nowak, Drying Technol. 31, 872 (2013).

[12] J. van Brakel, in: Advances in Drying, Ed. A.S. Mujumdar, Hemisphere, New York 1980, p. 217.

[13] G.W. Scherer, J. Am. Ceram. Soc. 73, 3 (1990).

[14] E.U. Schlünder, Drying Technol. 22, 1517 (2004).

[15] A.V. Luikov, Int. J. Heat Mass Transf. 18, 1 (1975). 